\title{
Concentración por flotación de menas de oro-cobre con bajas recuperaciones de arsénico
}

\author{
L. Chacón*, F. Ruiz** y R. Zapico***
}

\begin{abstract}
Resumen El objetivo del presente trabajo es definir los reactivos y las condiciones apropiadas para obtener concentrados con alta ley de cobre y baja recuperación de arsénico, a partir de muestras de una mezcla con $80 \%$ en peso de una mena de oro, cobre y bismuto, y $20 \%$ en peso de otra mena rica en oro y cobre, pero con alta ley de arsénico, provenientes de los yacimientos El Valle y Carlés, ubicados en Asturias-España, y procesados en una planta de concentración. La presencia de arsénico en el concentrado flotado es penalizada por sus compradores. La principal conclusión del trabajo es que un ditiofosfato como único reactivo colector es el que logra alcanzar el objetivo planteado, lo que se comprobó con pruebas en la planta de concentración.
\end{abstract}

Palabras clave Flotación. Recuperación arsénico. Recuperación cobre. Colectores. Depresor.

\section{Flotation of gold-copper ores with minimal arsenic recovery}

\begin{abstract}
The present work evaluates the reagents and appropriate conditions in order to obtain flotation concentrates with high copper content and low arsenic recovery. The studied ore is a blend of $80 \% \mathrm{w} / \mathrm{w}$ El Valle ore and $20 \% \mathrm{w} / \mathrm{w}$ Carlés ore, this latter being rich in copper and arsenic content. Those ores are worked from two deposits located in Asturias - Spain and treated at a concentration plant nearby. The arsenic content in the floated concentrate is penalized by the buyer. The main conclusion from the study is that a dithiophosphate is the collector that provides the best results, what was confirmed at plant-scale.
\end{abstract}

Keywords Flotation. Arsenic recovery. Copper recovery. Collectors. Depressor.

\section{INTRODUCCIÓN}

La empresa "Río Narcea Gold Mines S.A." ubicada en el Principado de Asturias, España, extrae y procesa en su propia planta de concentración, menas de dos yacimientos a cielo abierto: $\mathrm{El}$ Valle y Carlés. Las menas contienen, en común, oro y minerales de cobre, junto con minerales de arsénico (Carlés) y bismuto (El Valle), cuya presencia en el concentrado (rico en cobre) de la etapa de flotación de su planta extractiva es penalizada por sus compradores. La mena del yacimiento El Valle es tipo arcillosa, y la de Carlés tipo skarn sin alterar, con una dureza en la escala de Mohs significativamente mayor que la primera.
La empresa decidió alimentar, a partir de Abril de 2002, su planta de concentración con una mezcla $80 \%$ y $20 \%$ en peso de las menas de El ValleCarlés, respectivamente. De allí nace el presente trabajo, en el que se realizan una serie de ensayos de flotación en celda de laboratorio, fijando como objetivo principal la definición de los reactivos más apropiados y sus dosis, que permitan disminuir la ley de arsénico en los concentrados obtenidos para esa mezcla, buscando siempre recuperar, en la espuma flotada, la mayor cantidad de cobre posible.

La mezcla $80 \%-20 \%$, respectivamente, El Valle-Carlés presenta los siguientes contenidos promedios en g/t: 4,5 a 7 de oro; 3.000 a 5.000 de cobre; 4.500 a 11.500 de arsénico y una presencia de bismuto poco significativa.

(*) Escuela de Ingeniería Metalúrgica y Ciencia de los Materiales, Universidad Central de Venezuela, Ciudad Universitaria, Los Chaguaramos, 1040 Caracas, Venezuela

(**) Rio Narcea Gold Mines S.A., Av. Llaniello 13-Bajo, 33860 Salas, Asturias, España.

(**) Departamento de Ciencia de los Materiales e Ingeniería Metalúrgica, Universidad de Oviedo, Campus de Mieres, 33600 Mieres, Asturias, España.E-mails: luisechaconr@yahoo.es, francisco.ruiz@rngm.es, rzapico@uniovi.es 
Sobre flotación de menas de cobre y de menas que contienen oro existen numerosos y variados artículos publicados en revistas científicas. Para la realización del presente trabajo se tomaron en cuenta artículos referidos al uso de reactivos apropiados para esas menas ${ }^{[1-6]}$, un artículo sobre extracción en concentrados de cobre con alto contenido de arsénico ${ }^{[7]}$, manuales comerciales de agentes químicos de flotación editados por fabricantes y proveedores de los mismos, y experiencias previas realizadas por los autores del presente trabajo en la propia planta de concentración, con muestras de las menas, en distintas épocas recientes.

Para alcanzar el objetivo del trabajo se encontraron muy pocos artículos: Rivera, Flores y Gonzále ${ }^{[8]}$ evalúan la influencia de los xantatos sobre la flotación del arsénico presente en residuos líquidos; Chong et al. ${ }^{[9]}$ presentan experiencias que definen mejoras en el circuito de flotación en la planta de concentración de la mina Placer Dome's Campbell de Canadá, buscando separar con reactivos apropiados el oro de la arsenopirita. Fornasiero, Fullston, Li y Ralston ${ }^{[10]}$, separaron selectivamente por flotación dos minerales arsénico- cuprosos de otros minerales sulfurosos, evaluando la influencia de los distintos parámetros que intervienen. También, se tomaron en cuenta investigaciones realizadas por Alguacil $^{[11}$ y 12] y Alonso $^{[12]}$, en las que estudiaron la extracción de cobre en disoluciones en medio sulfato, con técnicas, fundamentos y antecedentes similares al presente trabajo.

\section{MATERIALES Y PROCEDIMIENTO EXPERIMEN- TAL}

Se tomaron muestras representativas de los yacimientos El Valle y Carlés, las cuales fueron secadas, trituradas en trituradora de rodillos (a tamaños menores de $2 \mathrm{~mm}$ ), mezcladas y homogenizadas en una relación en peso de $80 \%$ de mineral de $\mathrm{El} \mathrm{Va-}$ lle y $20 \%$ de Carlés, obteniéndose muestras de alrededor de $1 \mathrm{Kg}$, que se molieron en húmedo con $60 \%$ de sólidos, en un molino de barras de $3 \mathrm{Kg}$ de capacidad, con velocidad de giro de 70 r.p.m., por un tiempo de $70 \mathrm{~min}$, suficiente para la obtención de un $\mathrm{d}_{80}$ de $75 \mu \mathrm{m}$. Este tiempo de molienda se estableció a partir de la realización de una serie de ensayos de molienda-clasificación. Cada producto de la molienda sería la alimentación de un ensayo de flotación en celda de laboratorio.

Los ensayos de flotación se realizaron utilizando un equipo Denver D12, operando a 1.500 r.p.m., en las etapas de desbaste (rougher) y apure (scaven- ger); y 1.100 r.p.m. en las de limpieza (clean), usando celdas con 2,61 de capacidad para las dos primeras etapas, y 11 para las de limpieza. En cada ensayo, se repulpó cada muestra de $1 \mathrm{Kg}$ con el agua usada en el proceso de la planta extractiva. En cada ensayo, se tomaron los productos concentrados en cada etapa de la flotación y el estéril no flotado. Los productos fueron secados en estufa, pesados y llevados al laboratorio de análisis químico de la empresa, donde se definieron los contenidos de cobre y arsénico, con la técnica de Absorción Atómica de llama, y de oro por fusión y copelación.

La primera serie de ensayos consistió en probar una serie de colectores orgánicos, propicios para la concentración por flotación de minerales sulfurosos de cobre y de oro, representantes cada uno de los siguientes grupos de colectores: 1) Xantato (Xant.), 2) ditiofosfato (Dit.), 3) mezcla de mercaptobenzotiazol (Merc.) más ditiofosfato, 4) ditiofosfato más tionocarbamato (Tion.), 5) ditiofosfato más tionocarbamato y alcoholes (Alc.), 6) ditiofosfato más ditiofosfinato (Ditf.) y 7) tionocarbamato más alcoholes. La serie de ensayos se realizó siguiendo la siguiente secuencia: primer acondicionamiento (etapa en la que se añade el colector), seguido de dos etapas de desbaste, más segundo acondicionamiento $y$, una etapa de apure, con las mismas dosis de colector en el primer y segundo acondicionamiento, o sea, $30 \mathrm{~g} / \mathrm{t}$.

A continuación, se realizó una segunda serie de ensayos con el colector de la serie anterior que permitió alcanzar las menores recuperaciones de arsénico con una alta recuperación de cobre, agregando además un reactivo depresor o un dispersante con un acondicionamiento de $1 \mathrm{~min}$, previo a la adición del colector. Los depresores usados son eficientes para deprimir minerales no deseables en menas ricas en cobre y habían funcionado bien con las menas de El Valle y Carlés, por separado, en pruebas previas realizadas por los autores, o sea: $\mathrm{KMnO}_{4}$ y Gempolym GS3 (polisacárido modificado). El dispersante usado fue el $\mathrm{Na}_{2} \mathrm{SiO}_{3}$. La secuencia seguida fue: primer acondicionamiento (con el depresor o el dispersante); más segundo acondicionamiento (con el colector y el espumante), más dos etapas de desbaste; tercer acondicionamiento, más una etapa de apure, culminando con una etapa de limpieza. Las dosis añadidas fueron: $30 \mathrm{~g} / \mathrm{t}+15 \mathrm{~g} / \mathrm{t}+$ $5 \mathrm{~g} / \mathrm{t}$ para el colector solo y $300 \mathrm{~g} / \mathrm{t}$ para cada uno de los tres depresores utilizados. Esta última cantidad se añadió tomando en cuenta experiencias previas, antes citadas, con las menas por separado. 
La tercera serie de pruebas consistió en probar el colector más selectivo de cobre frente al arsénico, con distintas dosis, para definir la más conveniente a ser utilizada en la planta de concentración, siguiendo la misma secuencia utilizada en la planta, o sea: primer acondicionamiento, más dos etapas de desbaste, luego, un segundo acondicionamiento, más una etapa de apure, más un tercer acondicionamiento, finalizando con dos etapas de limpieza.

Para comprobar la buena selectividad del cobre frente al arsénico de los dos reactivos que mejor lograron ese objetivo en los ensayos de laboratorio, se hicieron ensayos en la planta de concentración, en un periodo de $12 \mathrm{~d}$, en los que se alimentó la planta con la mezcla estudiada, introduciendo cada uno de los reactivos, por separado, en cabeza de la etapa de desbaste del circuito de flotación, en diferentes días intercalados, como únicos agentes de flotación, siguiendo una secuencia diferente por días y probando pequeñas y diferentes dosis. Se decidió hacer esto último al tener en cuenta los resultados de la tercera serie de ensayos en el laboratorio. Junto con cada reactivo colector se añadió en cabeza de desbaste MIBC, como agente espumante.

De los resultados del análisis químico en las fracciones muestreadas por turnos de la planta en el tanque de rebose de los hidrociclones (alimentación del circuito de flotación en la planta de concentración) y en el tanque de concentrado de flotación, se tomaron en cuenta los valores, en ppm., de cobre y arsénico, a partir de los cuales se efectuaron los cálculos que permitieron definir las gráficas de resultados que se presentan en el siguiente apartado.

\section{RESULTADOS Y DISCUSIÓN}

\subsection{Ensayos en el laboratorio}

\subsubsection{Con reactivos colectores}

En todos los casos, se añadió la misma dosis en los acondicionamientos de las etapas de desbaste y apure: $30 \mathrm{~g} / \mathrm{t}$. En la figura 1, se representa la comparación entre las recuperaciones del cobre frente al arsénico obtenidas con los colectores empleados. Los tres puntos de cada curva representan, de izquierda a derecha, las recuperaciones que se acumularon en el orden de las etapas de la flotación: desbaste 1 , desbaste 2 y apure.

Se observa que, mientras el rango de recuperaciones de cobre es similar para todos los ensayos,

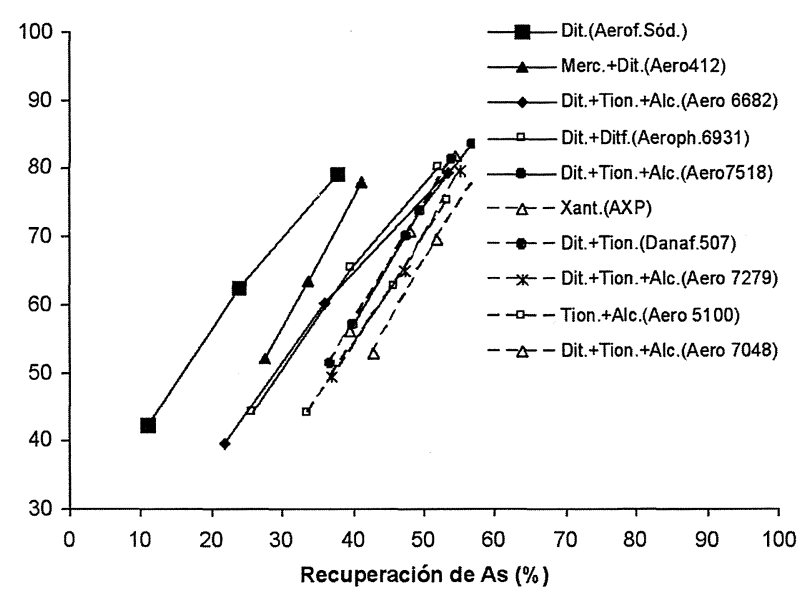

Figura 1. Resultados de las flotaciones con distintos colectores.

Figure 1. Results of the flotations with different collectors.

entre 40 y $85 \%$, las recuperaciones de arsénico son visiblemente más bajas cuando se añadió el colector con ditiofosfato como componente único (fue el más selectivo de todos, con recuperaciones acumuladas entre $11 \%$ en desbaste 1 , y $38 \%$ en apure) y el colector compuesto de la mezcla mercatobenzotiazol+ditiofosfato (entre 28 y $41 \%$ ). En la leyenda de las curvas de la figura 1 se presenta el orden obtenido de recuperaciones de arsénico, de las más bajas a las más elevadas.

\subsubsection{Con reactivos depresores}

Los resultados para la mezcla estudiada se representan en la figura 2, donde las menores recuperaciones de arsénico se obtuvieron en el ensayo estándar (con el ditiofosfato, el Aerofloat Sódico) y las mayores de cobre, en el ensayo con $\mathrm{KMnO}_{4}$.

Estos resultados revelan que los reactivos utilizados en las dosis empleadas, no presentaron un efecto depresor al arsénico en la mezcla de minerales estudiada, lo cual difiere de lo obtenido en los ensayos previos con muestras representativas de las menas de El Valle y Carlés por separado. Tal vez, las dosis empleadas de los depresores no sean las más convenientes, por lo que se recomienda hacer pruebas con otras dosis.

\subsubsection{Para definir la dosis más conveniente con el reactivo más selectivo frente al arsénico}

Los resultados obtenidos se presentan en los gráficos de la figura 3 y son indicativos de las recuperaciones 


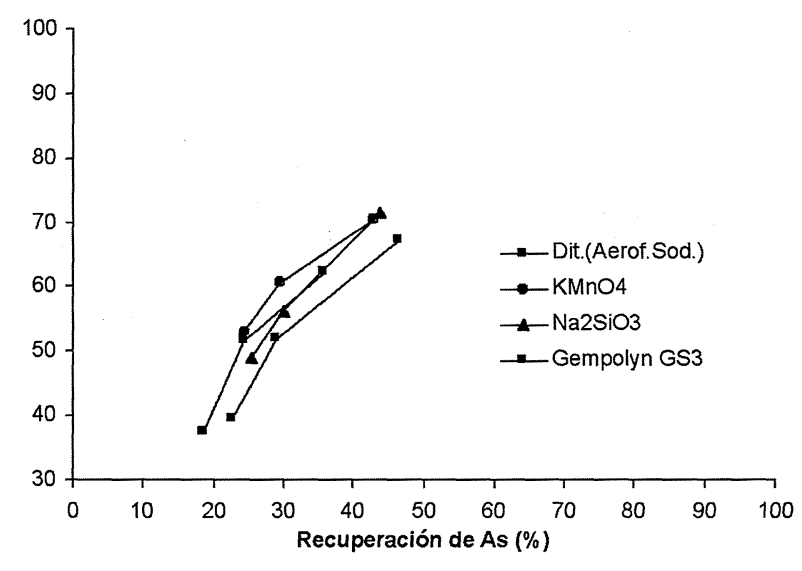

Figura 2. Resultados de pruebas con depresores y dispersantes comparando con Dit.

Figure 2. Results of tests with depressors and disperses comparing with Dit.
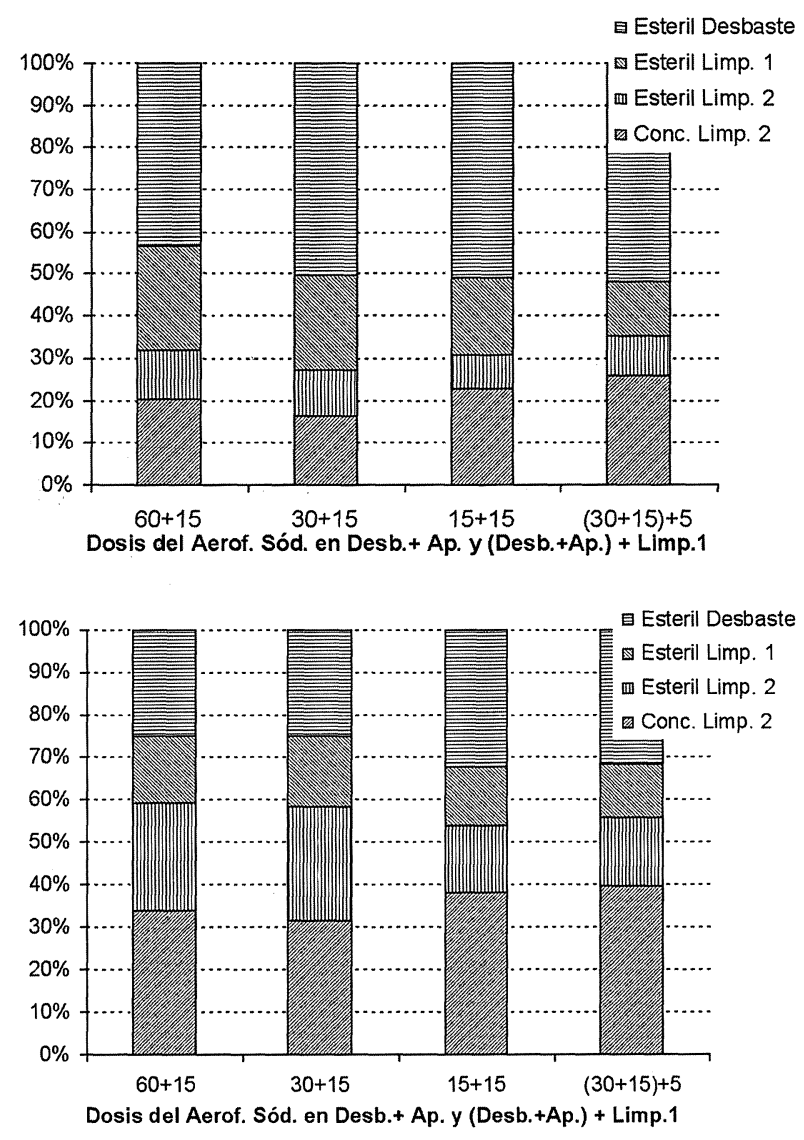

Figura 3. Resultados de los ensayos para definir la mejor dosis del mejor colector.

Figure 3. Results of test to define the best dose in the best collector.

de arsénico y cobre en cada producto de cada ensayo hecho con el ditiofosfato. Se observa que, la recuperación con la menor área en el concentrado final (el de la limpieza 2) se obtuvo para la dosis intermedia de $30+15 \mathrm{~g} / \mathrm{t}$, y la mayor recuperación de cobre, con la dosis más baja, de $15+15 \mathrm{~g} / \mathrm{t}$; mientras que, agregar una dosis mayor $(60+15$ g/t) y $5 \mathrm{~g} / \mathrm{t}$ previo a la primera limpieza no aportó mejores condiciones para lograr el objetivo del presente trabajo. Por tal motivo, se decidió utilizar bajas dosis del colector en la siguiente serie de ensayos.

\subsection{Ensayos en la planta de concentración}

Las pruebas se hicieron comparando el Aero 412 (merc.+ dit.) y Danafloat 123 (dit.), equivalente al Aerofloat Sódico. Los resultados del muestreo en la planta de concentración definen las gráficas de la figura 4, donde el rebose de los hidrociclones es la alimentación del circuito de flotación en la planta. Al compararlas, se puede concluir que las leyes de cobre son similares para ambos reactivos colectores, pero el ditiofosfato flota menos arsénico, de lo que se concluye que con el uso de éste reactivo en la flotación en la planta de concentración se ratifican los resultados obtenidos en los ensayos de laboratorio. Esto se obtuvo con dosis inferiores a $10 \mathrm{~g} / \mathrm{t}$, lo que permite asegurar que fue acertada la decisión tomada de usar bajas dosis de colector.
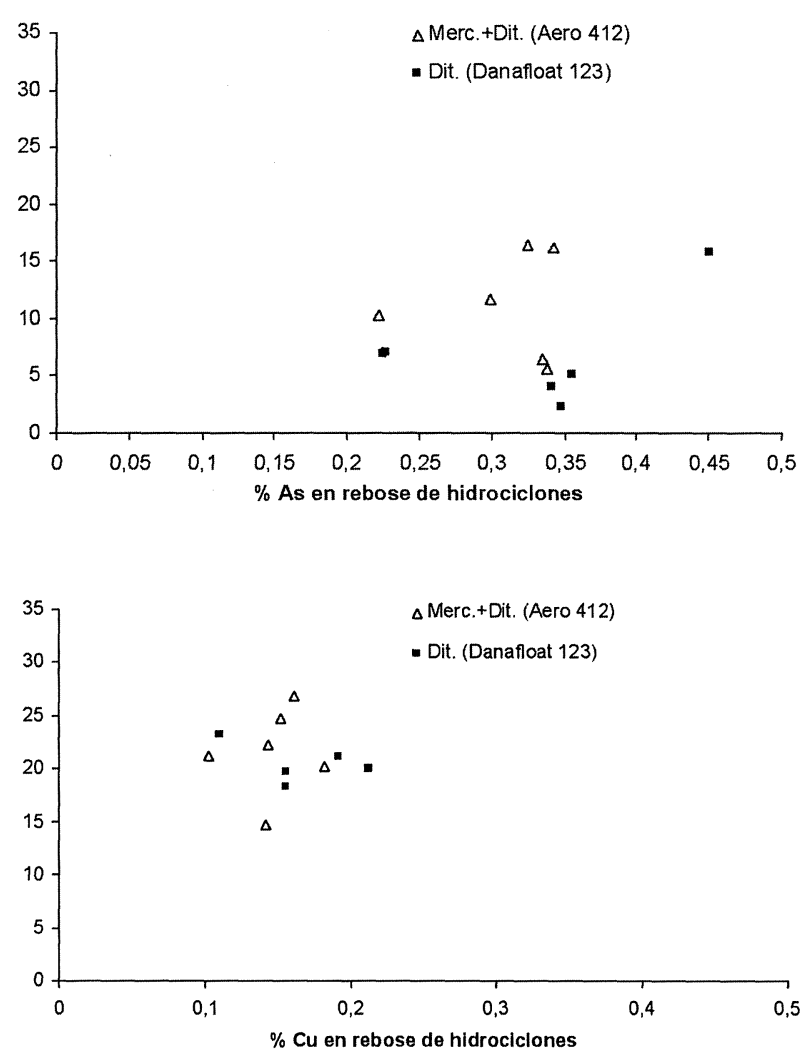

Figura 4. Resultados de los ensayos hechos en la planta de concentración.

Figure 4. Results of the test made in the concentration plant. 


\section{CONCLUSIONES}

- De colectores probados, el más conveniente para obtener concentrados con elevadas recuperaciones de cobre y bajas recuperaciones de arsénico, es aquel que contenga como componente principal el Ditiofosfato, con una dosis menor a $10 \mathrm{~g} / \mathrm{t}$.

- En las pruebas efectuadas con tres agentes depresor con las dosis empleadas, no se obtuvieron esperanzadores resultados en cuanto a la depresión del arsénico.

- Los ensayos realizados en la planta con el ditiofosfato comercial usado dieron muy buenos resultados, con recuperaciones de arsénico inferiores al $5 \%$ en el concentrado de flotación y leyes de cobre mayores del $18 \%$.

\section{REFERENCIAS}

[1] P.V. Avotins, S.S. Wang y D.R. Nagaraj, Reagents for BetterMetallurgy, SME Simposium proccedings, vol.1, Littleton; USA, 1994, pp. 47-55.
[2] D.R. NagaraJ, Trans. Indian Inst. Metall. 50 (5) (1997) 355-363.

[3] Z. EKMEMCi Y H. Demirel, Trans. Inst. Miner. Metall. 107 (1998) 76-81.

[4] P.K. Ackerman, G.H. Harris, R.R. Klimpel y F.F. Aplan, Miner. Metall. Process. 15 (1998) 27-35.

[5] R.R. Klimpel, Miner. Metall. Process. 16 (1999) 1-11.

[6] K. Forrest, D. YAN y R. DunNe, Miner. Eng. 14 (2001) 227-241.

[7] O. Herreros, G. Fuentes, R. Quiroz y J. Viñals, Rev. Metal. Madrid 39 (2003) 90-98.

[8] R.E. Rivera-Santillana, M. Flores y J.L. GonZÁlez, Afinidad 57 (2000) 279-282.

[9] T.Y. Chong, J.A. Folinsbee, A. Robitaille, T. Odo y G. WICKENS, CIM Bulletin. 92 (1999) 60-70.

[10] D. Fornasiero, D. Fullston, C. Li y J. Ralston, Intern. J. Miner. Process. 61 (2001) 109-119.

[11] F. J. AlguaCIL, Rev. Metal. Madrid 39 (2003) 205-209.

[12] F. J. Alguacil y M. Alonso, Rev. Metal. Madrid 38 (2002) 239-269. 